\title{
EL CAMPUS DE LA UNIVERSIDAD \\ DE CONCEPCIÓN: UN ESPACIO URBANO RELEVANTE PARA LA CONSTRUCCIÓN DE CIUDAD Y SOCIEDAD
}

Campus of the University of Concepción: Relevant urban space for the construction of city and society

\section{MARÍA DOLORES MUÑOZ*}

RESUMEN: En este artículo se plantea que el Campus de la Universidad de Concepción (Chile) es un espacio público relevante para la metrópolis de Concepción. Se presenta un enfoque interpretativo y un método basado en la gestión de archivos documentales. De esta manera, se desarrollan los conceptos de "campus" y de "espacio público", examinando los atributos físicos del conjunto universitario, singularidades, formas de inserción en la estructura urbana, aportes al proceso de construcción social de la ciudad y las relaciones con la trayectoria histórica de Concepción. Se cierra el artículo con reflexiones finales respecto a las implicancias del debate para entender el campus de la Universidad de Concepción como un espacio urbano relevante para la ciudad.

PALABRAS CLAVES: Campus, espacio público, Universidad de Concepción, valoración social, valor urbano.

ABSTRACT: The article proposes that the Campus of the University of Concepción (Chile) is a relevant public space for the Greater Metropolitan Area of Concepción. The study uses an interpretive approach and a method based on the analysis of key documents. The concepts of "campus" and "public space" are used to examine the physical attributes of the university complex, singularities, forms of insertion in the urban structure, contributions to the process of social construction of the city and relations with the historical trajectory of Concepción. The article closes with final reflections regarding the implications of the discussion to understand the University of Concepción campus as a relevant urban space for the city.

: Campus, public space, University of Concepción, social valuation, urban value.

Recibido: 20.05.2020 Aceptado: 1 1.07.2020

\section{KEY WORDS}

\footnotetext{
* Doctora en Arquitectura. Facultad de Arquitectura, Urbanismo y Geografía, Universidad de Concepción, Concepción, Chile marmunoz@udec.cl
} 


\section{INTRODUCCIÓN}

La historia de la Universidad de Concepción se inicia en 1917, cuando un grupo de profesionales liderados por Enrique Molina y Virginio Gómez formaron un comité para concebir una universidad, que se inauguró el 17 de marzo de 1919 con 123 estudiantes. Desde sus inicios, contó con el apoyo de personas, instituciones regionales y municipios. La fundación de la Universidad de Concepción representó una significativa innovación en la estructura educacional del país al disminuir la excesiva centralidad de la formación superior; también impulsó las dinámicas culturales de Concepción fortaleciendo las funciones urbanas relativas a la creación y difusión del conocimiento y la cultura. Por otra parte, la construcción del campus también es una referencia esencial en la historia urbana y social de la ciudad, porque rápidamente trascendió su función primaria como sede de la principal universidad regional, para constituir un espacio urbano altamente valorado por la comunidad penquista.

La fundación de la universidad es considerada la principal acción cultural del siglo XX (Campos, 1979) porque representó una significativa innovación en la estructura educacional del país al disminuir la excesiva centralidad de la formación superior; también impulsó las dinámicas culturales de Concepción fortaleciendo las funciones urbanas relativas a la creación y difusión del conocimiento y la cultura.

En 1919, Concepción se caracterizaba por la regularidad de su trama en damero, predominio de arquitectura neoclásica y construcciones de baja altura, sólo sobresalían los campanarios de las iglesias y el reloj del Liceo de Hombres. En estas circunstancias, el año 1917, el Dr. Virginio Gómez plantea la necesidad de construir un hospital moderno (García, 1994), que debía articularse con la creación de una Escuela de Medicina, vinculada a la futura Universidad, y que fue iniciado en 1940 con la construcción de un hospital de emergencia, ocupando dos edificios donde funcionaban las facultades de Leyes y Educación, para reemplazar al anterior hospital que había sido destruido por el terremoto de 1939. El nuevo recinto se inauguró el año 1943, originando un importante conjunto hospitalario, estructurado hasta hoy por el Hospital Clínico Regional y la Facultad de Medicina.

En su origen, el campus ocupaba un borde de Concepción, no obstante, a través del proceso de expansión de la ciudad y el crecimiento de la universidad se fue acentuando la integración del campus a la estructura urbana y fortaleciendo la relación espacial y funcional con varios lugares relevantes de la ciudad (Rodríguez y Carrasco, 2016; Torres, 20151. Los principales edificios universitarios son representativos de las tendencias predominantes en la arquitectura durante los últimos 100 años. En el campus conviven formas neoclásicas /fachada de la antigua Escuela Dental, destruida por el terremoto de 1960) con arquitectura moderna y ejemplos más recientes.

Así, el propósito de la investigación es analizar el valor social y urbano de la Universidad de Concepción como espacio público del área metropolitana de Concepción, reflexionando sobre los atributos, singularidades, estructura urbana, la vinculación con el medio y los procesos históricos del campus universitario.

\section{MARCO TEÓRICO}

La comprensión de las características físicas y sociales del Campus de la Universidad de Concepción está centrada en dos constructos interpretativos, por una parte, discusiones en torno a la categoría de campus universitario y, por otra, su condición de espacio público.

En su origen, los campus universitarios tendían a ocupar las periferias urbanas. Desde otro punto de vista, la noción de campus universitario se refiere a un territorio destinado al cultivo del conocimiento, y que, en el caso de la Universidad de Concepción, muestra 
influencias de la Universidad de California en Berkeley, producto de la visita de Enrique Molina a varias universidades estadounidenses (García, 1994) donde se promovía una visión innovadora sobre la conexión entre el paisaje y las estructuras físicas.
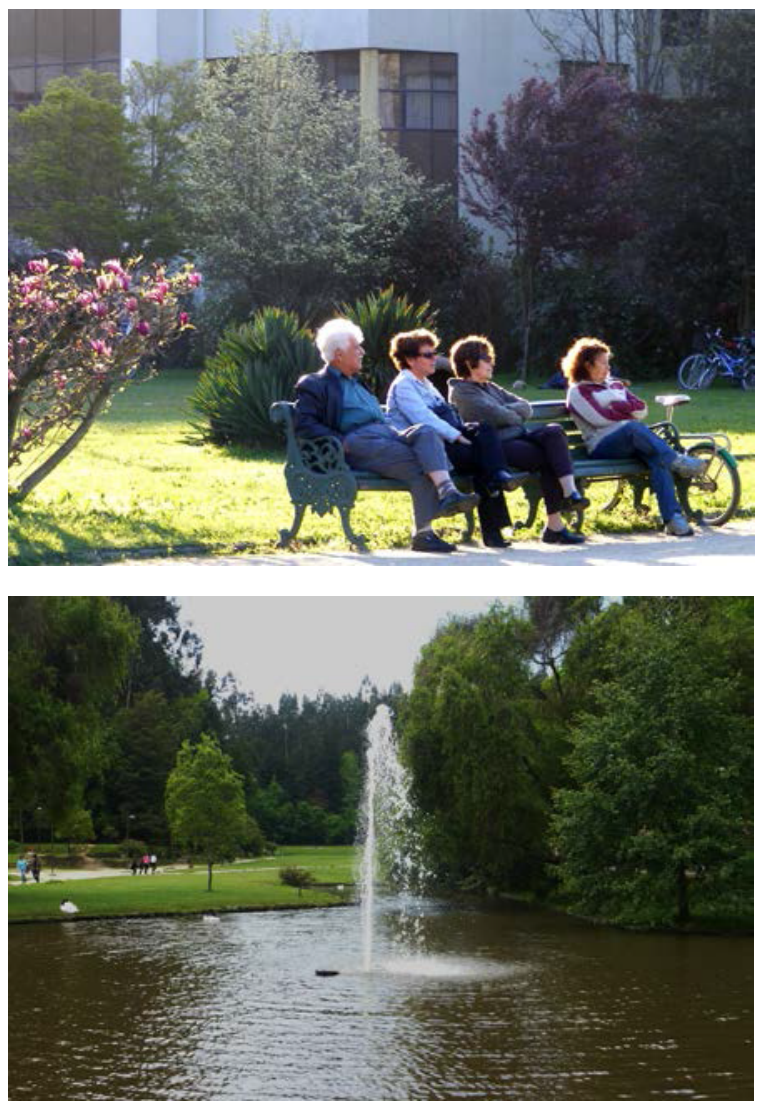

Figura 1. Jardines del campus. Fuente: Juan Pablo Bascur. Archivo fotográfico María Dolores Muñoz.

Respecto del campus como espacio público, es preciso señalar que, como explica Alguacil (2008), el espacio público siempre es un espacio colectivo, un lugar donde las personas pueden encontrarse e interactuar, esta condición se puede manifestar en un espacio de propiedad pública, pero también en espacios de dominio y uso público (ver figura 1). El dominio público se relaciona con un sentido cultural y el uso público con un sentido social. En Chile, la legislación no contiene referencias al espacio público, aunque entre los distintos tipos de bienes se identifican, entre otros, a los Bienes Nacionales de Uso Público (MINVU, 2009). No obstante, como indica Schlack (2007), la definición de Bien Nacional de Uso Público mezcla a la propiedad con el uso y, en este sentido, se debe considerar que la vida pública transcurre tanto en espacios públicos como en espacios privados, además, la sociología no diferencia a los lugares según su propiedad, sino de acuerdo a quién lo usa. Desde esta perspectiva, la característica distintiva del espacio público es su grado de accesibilidad o la posibilidad de uso para cualquier persona. Borja y Muxi (2003, p. 15) plantean que el espacio público es, a un tiempo, el espacio principal del urbanismo, de la cultura urbana y de la ciudadanía; es un espacio físico, simbólico y político que facilita el encuentro de los ciudadanos, permitiendo las manifestaciones políticas, culturales y sociales que dan significado a la vida urbana. Así, los espacios públicos son los lugares de intercambio y comunicación, son escenarios urbanos dilectos donde los ciudadanos pueden participar activamente en acontecimientos relevantes de la vida social, política y cultural de la ciudad. En sentido similar, Boria (2005) señala que el espacio público es un ámbito de relaciones y de cohesión social, es un referente que da sentido a la vida urbana, que marca simbólicamente el territorio y que proporciona elementos de identidad específica.

Por otra parte, los espacios públicos también son lugares donde se desarrollan actos cotidianos y prácticas habituales que, aun cuando, parecen no tener relevancia, son la base de vínculos portadores de identidad (Alessandri, 2014). Al respecto, Gehl (2006) señala que una condición irrenunciable del urbanismo es realizar la vida en sociedad mediante la humanización del espacio urbano, reforzando su función como espacio de encuentro. Así también, Lefebvre (1976) señala el derecho a encontrarse y reunirse, indicando que la ciudad debe responder a determinadas necesidades y funciones, como son la necesidad de vida social, función lúdica y función simbólica del espacio. En este sentido, se destacan aquellos lugares donde, además de las actividades que forzosamente se deben realizar, surgen actividades opcionales y sociales que se expresan en el juego, paseo y actos colectivos. 


\section{METODOLOGÍA}

La investigación propone una metodología de investigación documental, desarrollando diferentes etapas para acercarse al fenómeno del campus universitario y su relación con el espacio público (Bosch, 2008). El objeto de estudio es el campus de la Universidad de Concepción, ubicado en la ciudad de Concepción, región del Biobío, Chile. Objeto que es observado considerando las estructuras físicas del campus y su vinculación con la sociedad.

La recolección de la información se realizó a través de una etapa de búsqueda de fotografías y documentos oficiales de la Corporación de la Universidad de Concepción, Memoria Histórica y archivos fotográficos privados, desarrollando un tratamiento de los datos visuales en función de lineamientos del análisis documental (Gibbs, 2012). Para luego construir un discurso sobre la descripción del espacio público de la universidad y una interpretación teórica de las relaciones que enlazan a las estructuras físicas con las necesidades sociales, culturales y educativas del espacio público.

\section{RESULTADOS}

\section{Análisis del conjunto universitario}

Un rasgo destacado del campus es su alto valor de uso por parte de los ciudadanos, es un espacio urbano con acceso público, por esto, en el decreto que establece la condición patrimonial del campus se señala que desde "una perspectiva integral, con un sentido altamente cívico, favoreció su apropiación y pertenencia no solo de la comunidad universitaria, sino que de todos los ciudadanos, siendo parte de la memoria social y la vida cotidiana de los penquistas, constituyéndose de esta manera como un hito de la ciudad" (Decreto 393, 2016 , p.3). La importancia urbana del campus se relaciona con su dimensión física y su dimensión social, por esto, se expresa en la forma y disposición de los edificios, jardines y recorridos, pero también, en el uso de los lugares y de sus símbolos. La dimensión física concierne al aporte del campus a la construcción material de la ciudad y está representada por la arquitectura, el paisaje urbano, senderos, árboles y obras de arte. La dimensión social se manifiesta en lugares de encuentro, en las expresiones culturales que se despliegan en el campus y en las funciones simbólicas del espacio; esta dimensión atañe a la formación de ciudadanos y, por lo tanto, a la construcción de sociedad. El campus es valorado por su arquitectura, sus espacios abiertos, jardines, monumentos y su historia, que transcurre en paralelo a la historia de la ciudad.

El conjunto de construcciones que se concentran en el campus revela las transformaciones históricas y urbanas de la ciudad. Además, muestran los cambios de la arquitectura chilena desde las primeras décadas del siglo XX. Varias de estas piezas arquitectónicas forman parte del Eje Central, un espacio destacado por sus proporciones y valor patrimonial, declarado Monumento Nacional en categoría de Monumento Histórico (Decreto 393, 2016) (Ver figura 2). Este eje comienza en el Arco de la Universidad, la puerta monumental del ámbito universitario, conocida como Arco de Medicina y que fue sede de la Facultad de Medicina, y termina en la Biblioteca Central, diseñada en la década de los 70 por Emilio Duhart y Roberto Goycoolea, de acuerdo con los principios compositivos y funcionales de la arquitectura moderna. 


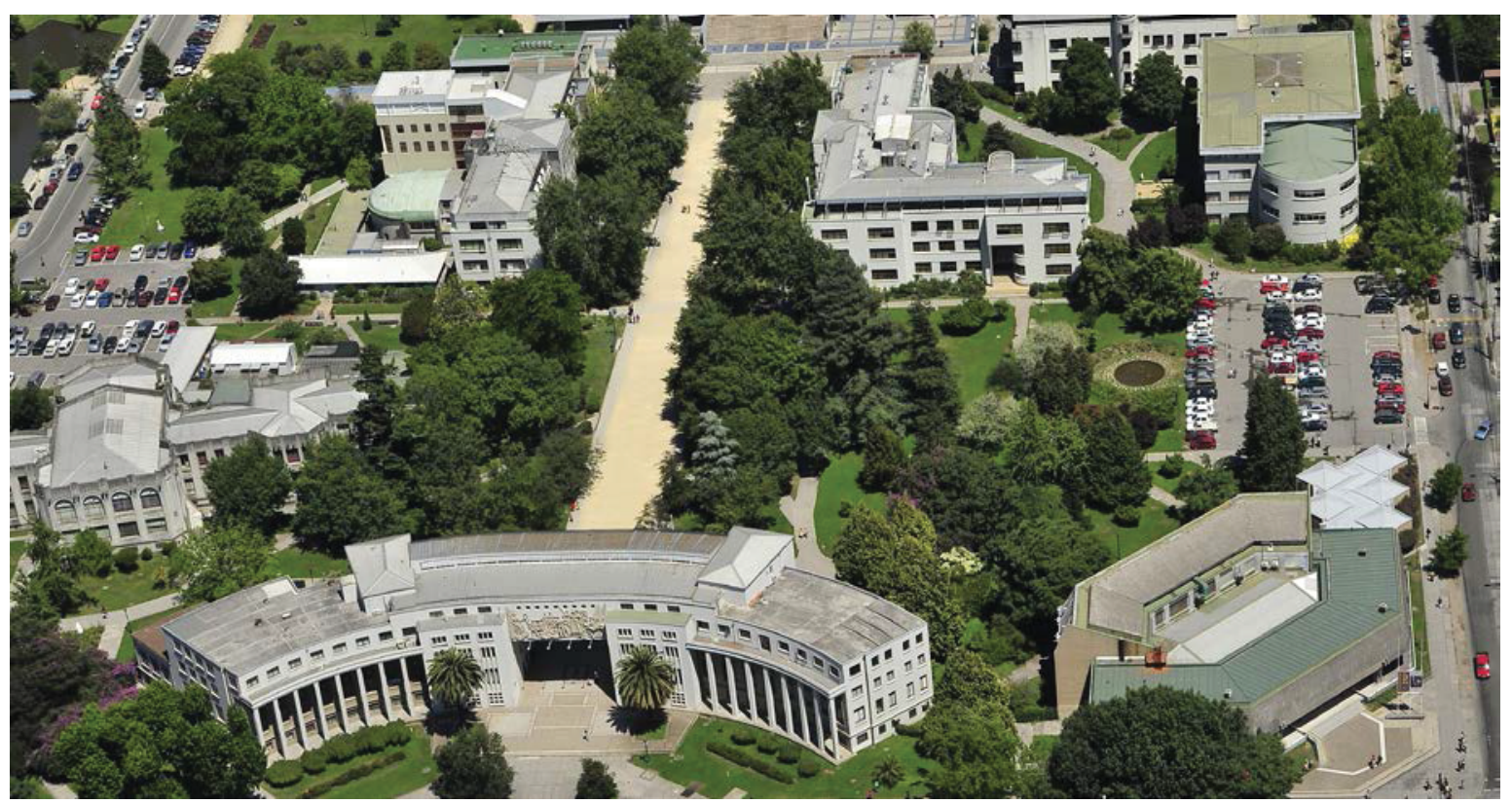

Figura 2. Vista aérea del Eje Central con Arco de Medicina. Fuente: Archivo fotográfico María Dolores Muñoz.

El Arco de la Universidad (ver figura 3) se destaca por forma, proporciones y columnata monumental. El conjunto integrado por las Facultades de Farmacia y Ciencias Naturales y la Facultad de Humanidades (antiguo Instituto de Lenguas) es representativo de la arquitectura moderna en su fase inicial.
La arquitectura racionalista, inspirada en nociones compositivas de la Bauhaus, tiene referentes en la Casa del Deporte y en la residencia del primer rector Enrique Molina, actual edificio de la Rectoría. La arquitectura con rasgos Art Decó está representada por el Instituto de Anatomía (Ver figura 4) y la Facultad de Leyes (ver figura 5).

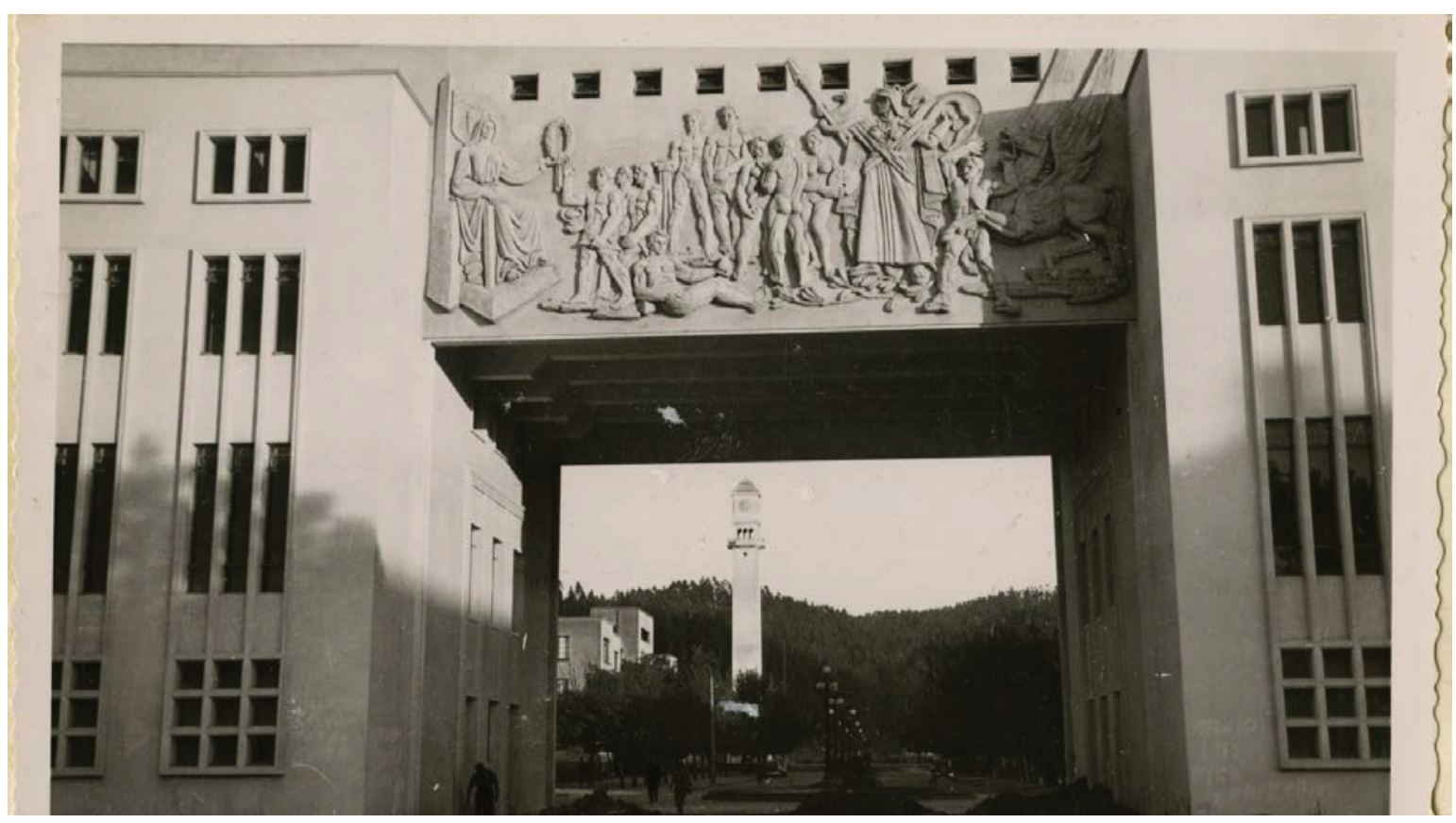

Figura 3. Arco de Medicina, Campanil. Fuente: Archivo fotográfico Universidad de Concepción, Concepción, Chile. 


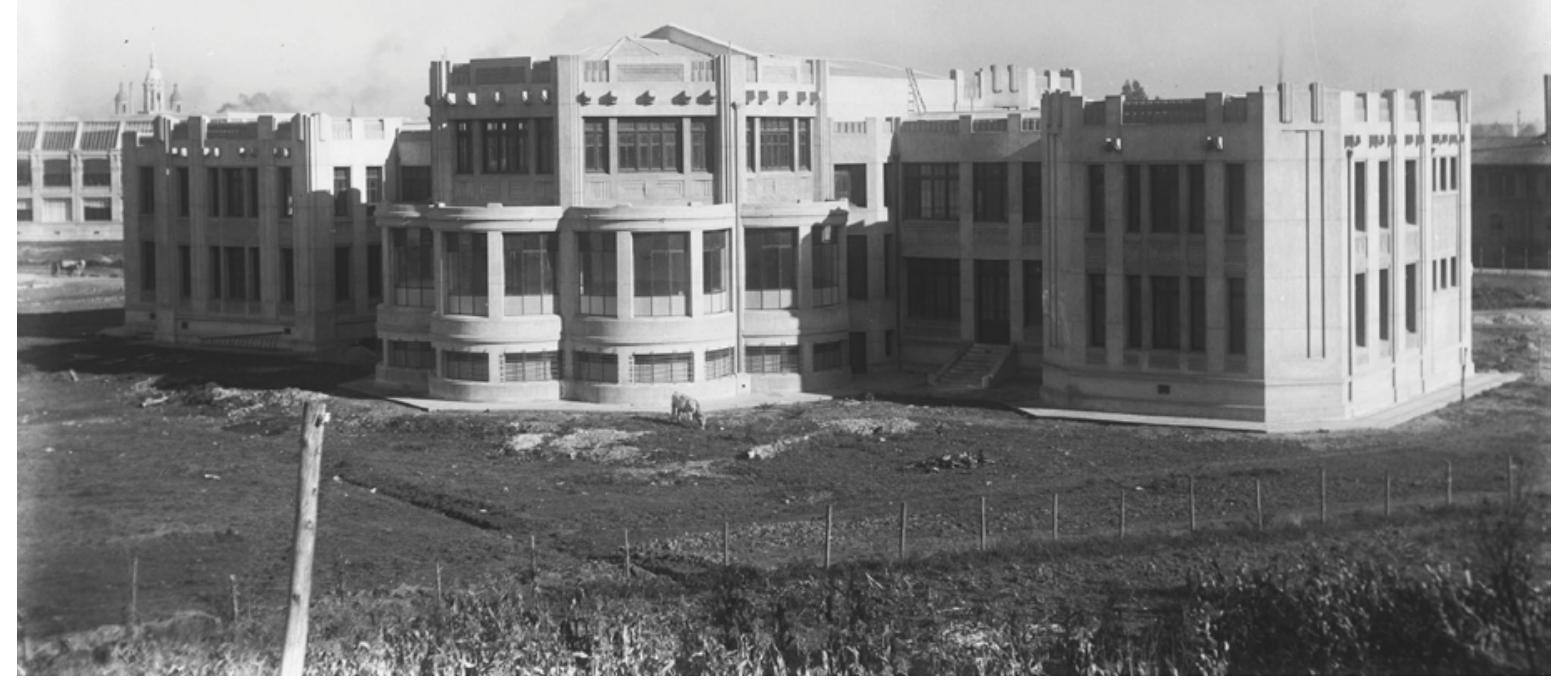

Figura 4. Instituto de Anatomía. Fuente: Archivo fotográfico Universidad de Concepción, Concepción, Chile.

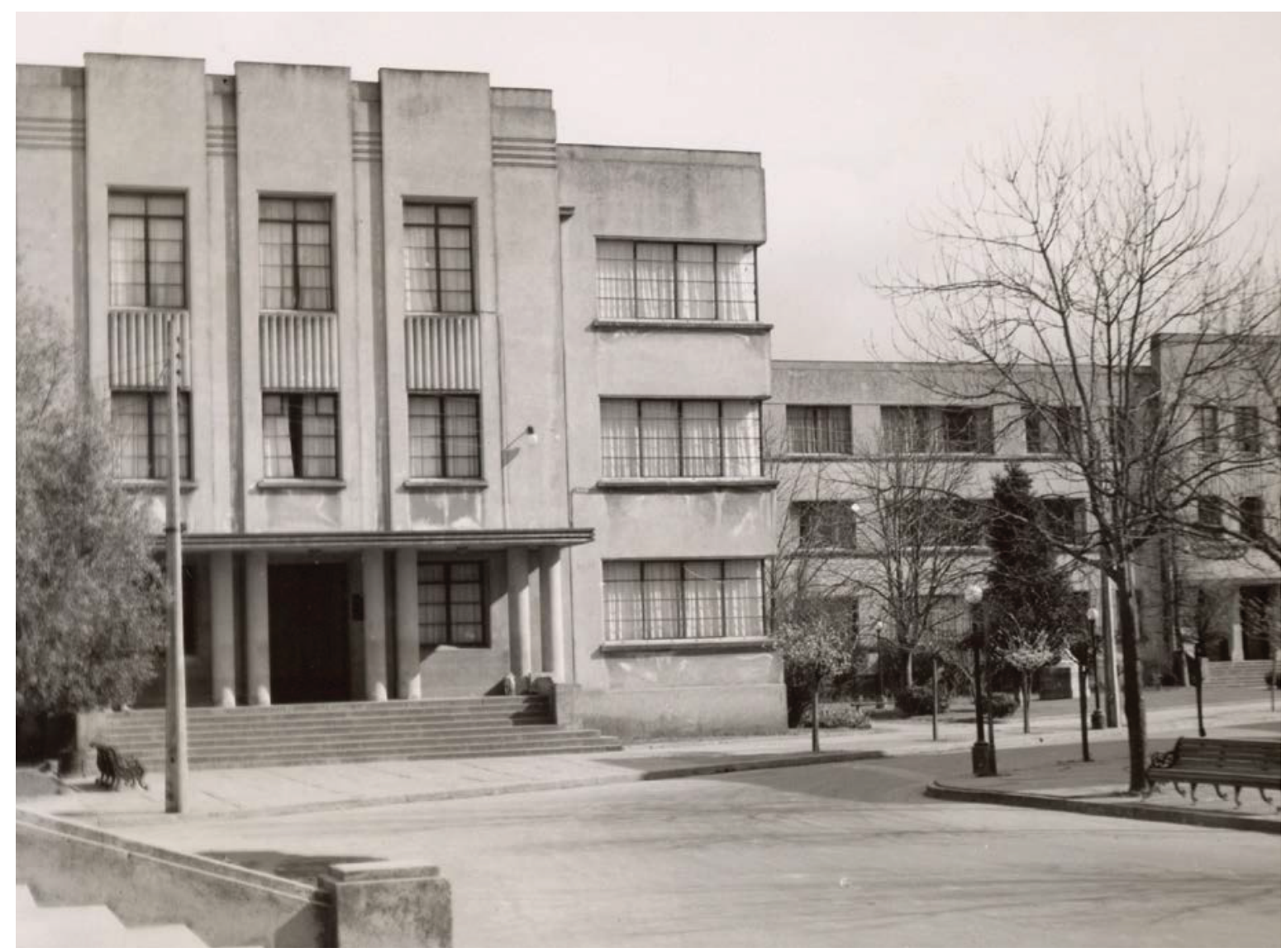

Figura 5. Escuela de Leyes y Facultad de Humanidades (antiguo Instituto de Lenguas). Fuente: Archivo fotográfico Universidad de Concepción, Concepción, Chile. 
La arquitectura característica de las primeras décadas del siglo XX conforma el sector más antiguo del campus, vinculado a las propuestas urbanas de Carlos Miranda y Karl Brunner. Un segundo sector, perfilado en base a la arquitectura moderna de mediados del siglo XX, está organizado en torno al Foro abierto y los edificios de la Facultad de Ingeniería, diseñados por Emilio Duhart, subrayando la hegemonía del espacio como protagonista del orden urbano, cualidad que se destaca mediante una arquitectura de volumetría abstracta y regida por una modulación ortogonal (ver figura 6). El límite entre los sectores históricos del campus está señalado por el Campanil, torre de $45 \mathrm{~m}$ de altura, considerado el principal símbolo de la universidad y de la ciudad de Concepción. El Foro abierto es el lugar protagonista del Eje Central y el espacio de interrelación entre el sector más antiguo y el intermedio. La Biblioteca Central es otro límite entre realidades urbanísticas diferentes.

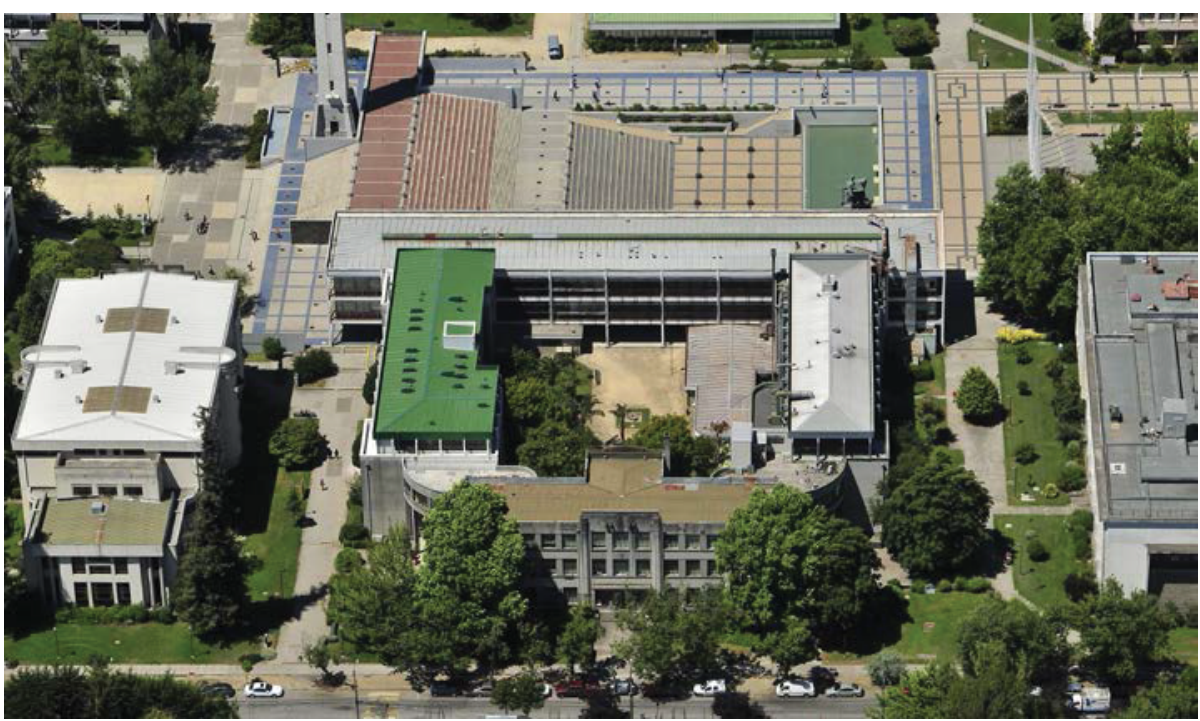

Figura 6. Foro Abierto de la Universidad de Concepción, Concepción, Chile. Fuente: Archivo fotográfico María Dolores Muñoz.

No obstante, a pesar de la variedad y predominio de edificios que no se relacionan por semejanzas morfológicas o constructivas -aunque tienen alturas similares - el campus mantiene su integridad por la existencia de amplios vacíos que hacen posible el dominio visual del espacio y la lectura individual de los volúmenes. La característica de ámbito cohesionado destaca a los sectores comprendidos entre el Arco de la Universidad y la Biblioteca Central, no deriva de la arquitectura sino de la potencia ordenadora del Eje Central, que articula a los recorridos y edificios que lo integran (ver figura 7).

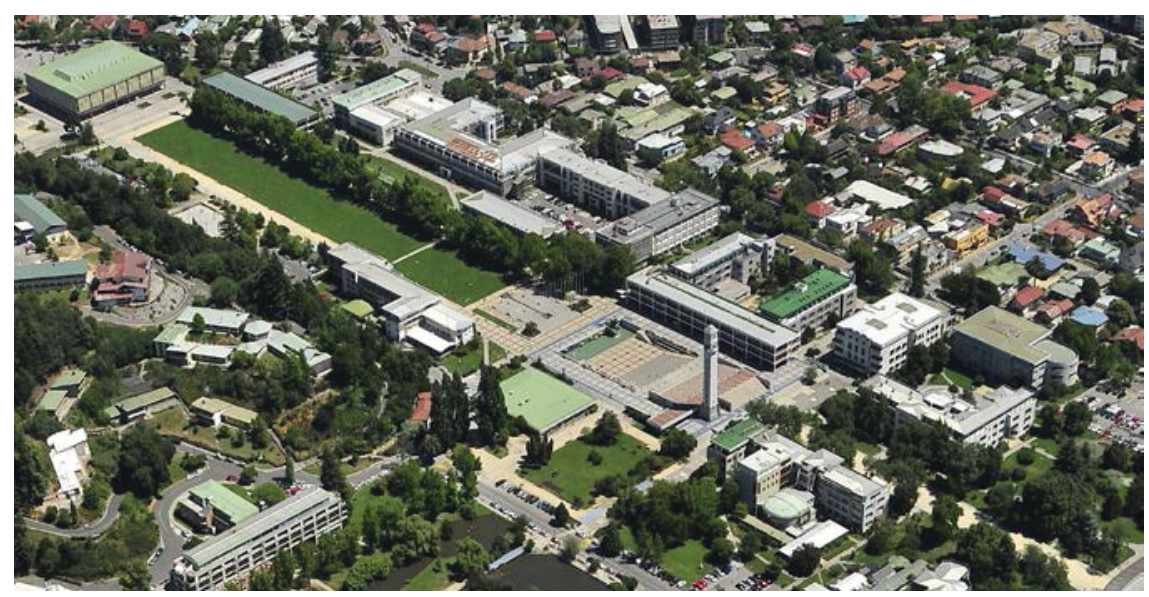

Figura 7. (Foto 9) Eje Central moderno. Fuente: Archivo fotográfico María Dolores Muñoz. 
Análisis de las relaciones con la trama urbana y el crecimiento de la ciudad.

En el campus se han llevado a cabo varias intervenciones que expresan la diversidad que caracteriza a la arquitectura actual. Un hito de los cambios, ocurridos a partir de 1970, fue la construcción del edificio de Aulas, con una planta circular que revela una intención de autonomía respecto al entorno. Así, la distribución de las construcciones y distintas soluciones morfológicas fueron segregando al campus en varios dominios territoriales.

En el contexto urbano, la construcción del campus representó el surgimiento de un paisaje diferente porque respondía a una concepción urbanística innovadora que prescindió del trazado cuadricular dominante en la ciudad. La ocupación del espacio universitario se organizó a partir de áreas verdes y ejes funcionales y visuales que resaltan a los edificios como volúmenes aislados y privilegian la condición de espectador de quien recorre el lugar. La novedad que proclamaba el diseño del campus puede interpretarse como un reconocimiento a la nueva realidad cultural que emergía en la ciudad o como expresión de libertad que remite a la autonomía académica, al debate pluralista y al lema de la Universidad de Concepción: por el desarrollo libre del espíritu.

Por su organización espacial, el campus se diferencia nítidamente del trazado cuadricular, al mismo tiempo, refuerza su relación de inmediación con el contexto cercano porque el eje principal del campus es una prolongación de la calle Janequeo y las principales diagonales de Concepción tienen uno de sus extremos en edificios universitarios. La diagonal Pedro Aguirre transcurre desde la Plaza de Los Tribunales hasta la Plaza Perú y remata en la Pinacoteca; la diagonal Roosevelt conecta a la Casa del Deporte con la Plaza Juan Bosco; además, los ejes transversales del campus prolongan el trazado de las calles adyacentes. Así, aunque el campus tiene una estructura urbana propia y distintiva, sus líneas principales se integran a la ciudad constituyendo prolongaciones de la trama urbana (ver figura 8).

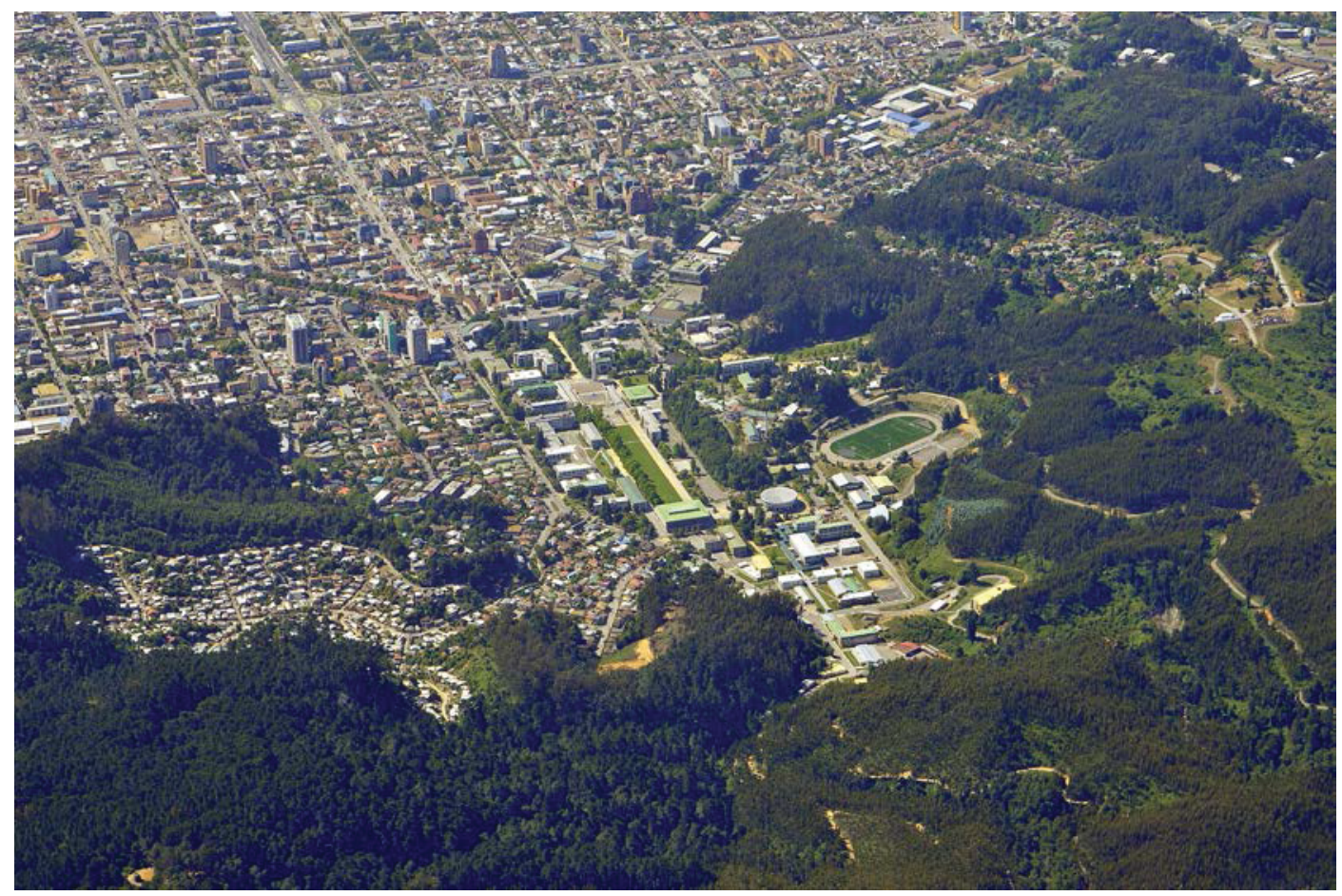

Figura 8. Integración del campus en la estructura urbana de Concepción, Chile. Fuente: Archivo fotográfico María Dolores Muñoz. 
Análisis del uso de los espacios del campus

Por otra parte, la construcción del campus también es una referencia esencial en la historia urbana y social de la ciudad, porque rápidamente trascendió su función primaria como sede de la principal universidad regional. El campus de la Universidad de Concepción, por su condición de espacio urbano, puede ser analizado destacando sus cualidades físicas, culturales o estéticas. Además, de sus funciones propias, vinculadas a la formación profesional y a la generación y trasmisión de conocimiento, el campus enriquece la estructura urbana, aporta una extensa superficie verde con valiosas especies arbóreas, contribuye al desarrollo cultural mediante la exposición de diversas expresiones de arte.

La importancia del campus, como ámbito irradiador de cultura, se expresa nítidamente en el Foro abierto, donde se integran las relaciones funcionales, visuales y espaciales que enlazan a la estructura urbana, los edificios, jardines y obras de arte. Con su anfiteatro, gradas y el respaldo del monumento, homenaje al espíritu de los fundadores de la Universidad de Concepción, el Foro funciona como una plaza cívica que realza el doble carácter del campus que, a la vez, es recinto universitario y espacio público.

El Foro puede describirse como un lugar colmado de valores culturales y donde sus funciones y significados actuales se amplían con valores simbólicos heredados del urbanismo clásico como aquellos arraigados al fórum, principal espacio de encuentro cívico de las ciudades latinas. También, esta unidad se integra al Campanil, otro elemento provisto de reconocidos significados culturales y urbanos, promoviendo un magnífico escenario al aire libre, donde la comunidad universitaria y los ciudadanos pueden asistir a conciertos, presentaciones de danza y teatro (ver figura 9).

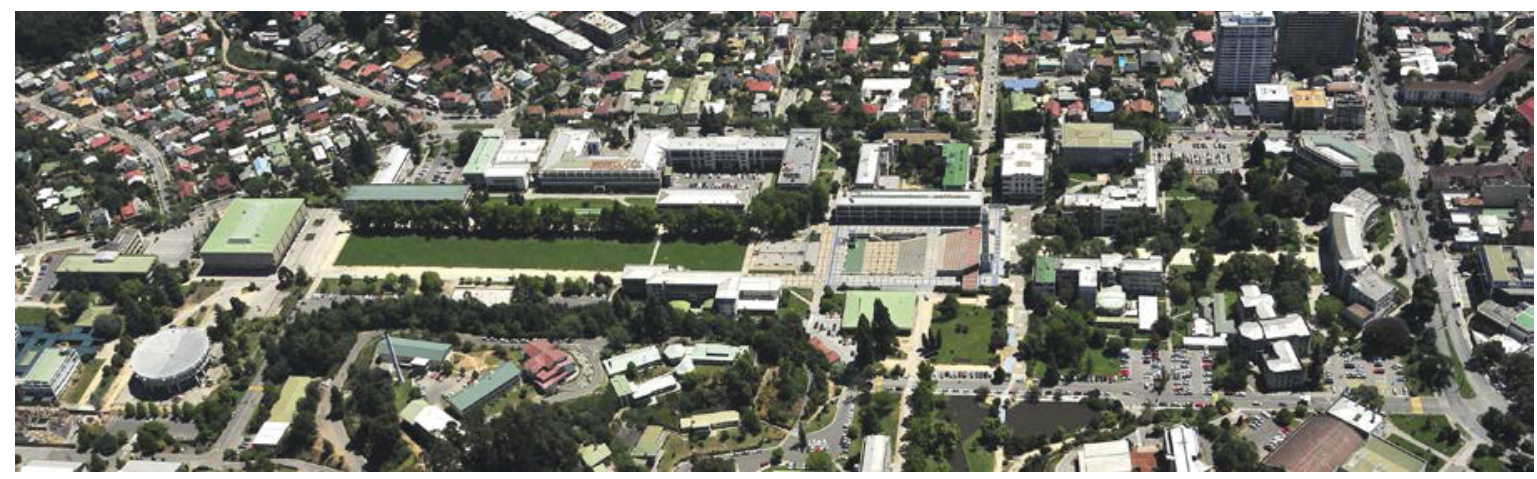

Figura 9. Vista del Foro durante su construcción. Fuente: Archivo fotográfico Universidad de Concepción, Concepción, Chile

La condición de espacio público del campus no está presente sólo en el Foro, porque esta característica impregna a la totalidad del campus y debe resaltarse, ya que en el espacio público radican los valores básicos de una ciudad. El reconocimiento del campus como espacio importante para el público explica por qué, en marzo del año 2000, el Foro abierto fue elegido por el presidente Ricardo Lagos como un escenario urbano del cambio de mando presidencial.

El campus es el espacio donde transcurre la vida universitaria y se celebra la vida ciudadana, porque cotidianamente los estudiantes y profesores se desplazan a las facultades, aulas, bibliotecas y laboratorios. Estos recorridos, se relacionan con actividades académicas obligatorias y se enlazan con otras actividades opcionales y sociales originadas por la existencia de espacios que potencian diversas formas y posibilidades de encuentro social (ver figura 10).

En primavera, los prados del campus se convierten en lugares de estudio al aire libre 
y en las tardes de los viernes el Foro se activa como espacio social con sus graderías atestadas de estudiantes esperando el inicio de un recital de música. En verano y los fines de semana el campus se transforma en un lugar para el juego y el deambular, demostrando su capacidad como espacio social y que se muestra con orgullo a los visitantes, siendo el escenario urbano predilecto para que los novios obtengan sus fotografías.
Los niños se embelesan con los cisnes y patos de la laguna o recorren en bicicleta los senderos rodeados de flores, los ancianos pasean olvidando las estridencias urbanas, los jóvenes conversan y leen amparados por la sombra de los árboles o se besan en la privacidad de los jardines más recónditos. Así, día tras día, la vida en el campus se va imprimiendo, como una huella imborrable en la memoria de los estudiantes, los profesores y los ciudadanos para formar parte de sus recuerdos más preciosos.

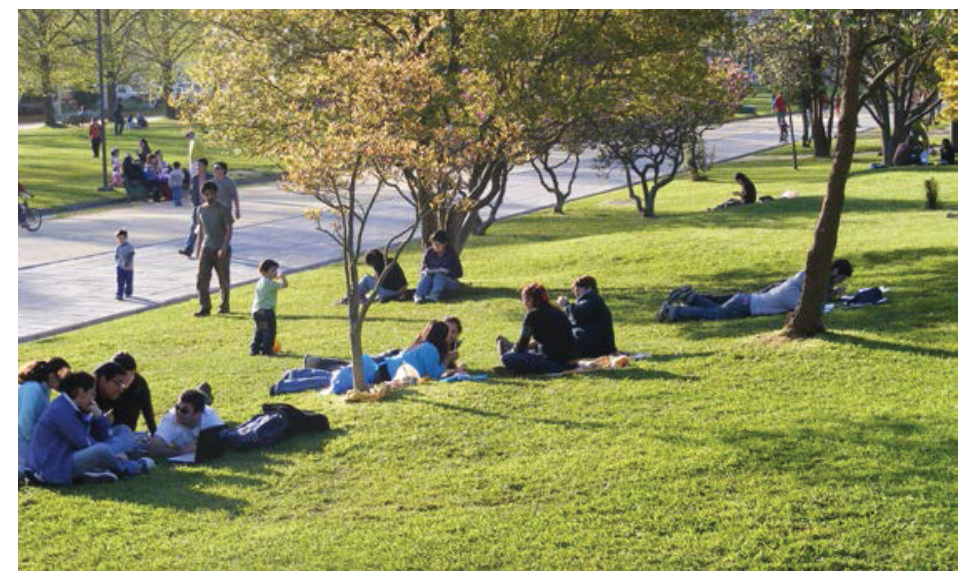

Figura 10. El campus como espacio de encuentro. Fuente: Juan Pablo Bascur. Archivo fotográfico María Dolores Muñoz.

\section{DISCUSIÓN Y CONCLUSIONES}

Al analizar la morfología y los usos del campus se promueve una comprensión del campus de la Universidad de Concepción como: a) un espacio con acceso público con una alta valoración social que revela los procesos de transformación histórica del área metropolitana Concepción; b) un espacio que representa la diversidad de la arquitectura del siglo XX, evocando el surgimiento de paisajes urbanos innovadores que prescindieron del trazado cuadricular, organizando el espacio desde áreas verdes y ejes visuales funcionales c) y, un espacio que trascendió su función educativa, para transformarse en un promotor de cultura y de vinculación con la sociedad local, regional y nacional.

El campus de la Universidad de Concepción es uno de los principales espacios urbanos de Chile. Es socialmente valorado por sus características distintivas, su condición de patrimonio cultural que fortalece la identidad colectiva y por constituir un lugar de vinculación afectiva con el territorio y el paisaje (Muñoz, 1995). En la situación específica de la Universidad de Concepción, el campus, además de ser un ámbito destinado a cultivar el conocimiento, permite incorporar a la naturaleza cultivada en la estructura urbana, porque fue concebido según los principios morfológicos de la ciudad jardín, de modo que los edificios se vinculan mediante avenidas arboladas y amplios jardines configurando un paisaje donde la vegetación y el agua aportan belleza y calidad ambiental. El protagonismo de la naturaleza permite que el campus constituya un encadenamiento verde con el Parque Ecuador y se integre al sistema del Cerro Caracol, principal envolvente natural de Concepción.

Además, la investigación señala la importancia de los símbolos vinculados a la comunidad penquista y que se asocian a lo señalado por Borja y Muxi (2003), en 
donde la cultura y el urbanismo encuentran una relación, provocando un encuentro entre la ciudadanía y el espacio público, que presenta características únicas a nivel nacional y que desarrollan la identidad de los habitantes (Alessandri, 2014).

El campus tiene una extensión que lo destaca como espacio urbano, pero, a la vez, reconoce la medida del hombre porque puede ser atravesado caminando, pausadamente, en pocos minutos. Por su posición dentro de la trama urbana de Concepción, es un lugar cercano al corazón de la ciudad -centrado en la Plaza de la Independencia- y, al mismo tiempo, mantiene su carácter de ámbito retirado de la vorágine urbana donde se potencia la contemplación creativa, el diálogo y la conversación. El campus, como expresión física de la Universidad de Concepción, históricamente ha sido ejemplo de buenas prácticas urbanas. Por esto, la planificación del campus debiera ser una oportunidad para construir espacios y edificios que puedan orientar a las futuras acciones urbanísticas en la ciudad, reforzando el protagonismo universitario para impulsar el desarrollo urbano y social.

El último resultado evidencia que la Universidad de Concepción, como componente del entramado urbano, responde a las necesidades de la vida y las funciones sociales presentadas por Lefebvre (1976). Por ende, el campus es representando como un lugar que potencia las relaciones y los vínculos entre la comunidad penquista al permitir la realización de actividades culturales, económicas, políticas y patrimoniales, entre otras.

El campus de la Universidad de Concepción es un lugar donde se promueve la convivencia social, constituyendo un paradigma de espacio público que acoge al ciudadano. Las nuevas demandas sociales, como la equidad de género y valoración de la diversidad social, exigen universidades competitivas, no sólo en lo concerniente al prestigio académico, sino como contribución al país y la región mediante la formación integral de los estudiantes como profesionales calificados y ciudadanos socialmente responsables. En este contexto, el campus debe reforzar su cualidad de espacio público que promueve el intercambio social y la identificación simbólica con la cultura propia, así, la universidad construye ciudad y construye sociedad.

\section{REFERENCIAS BIBLIOGRÁFICAS}

Alessandri, A. (2014). La ciudad como privación y la reapropiación de lo urbano como ejercicio de la ciudadanía. Scripta Nova, 493(08), 1-14. Recuperado de https://revistes.ub.edu/index.php/ ScriptaNova/article/view/ 14979

Alguacil,J. (2008). Espacio público y espacio político. La ciudad como el lugar para las estrategias de participación. Polis, 7(20), 199-223.https://scielo.conicyt.cl/pdf/ polis/v7n20/art 1 1.pdf

Bosch, C. (2008). La técnica de investigación documental. México: Trillas.

Borja, J. (2005). Urbanismo y ciudadanía. Los Monográficos de Barcelona Metrópolis del Mediterráneo. $N^{\circ} 6$ Civismo: Las Claves de la Convivencia, 43-50.

Borja, J. y Muxi, Z. (2003). El espacio público: ciudad y ciudadanía. Barcelona: Electa.

Campos, F. (1979). Historia de Concepción. 1550-1970. Santiago: Editorial Universitaria.

Decreto 393 (2016). Declara Monumento Nacional en la categoría de Monumento Histórico al "Campus Central de la Universidad de Concepción". Recuperado de https:// www.monumentos.gob.cl/monumentos/ monumentos-historicos/campus-centraluniversidad-concepcion 
García, J. (1994). El Campus de la Universidad de Concepción, su desarrollo urbanístico y arquitectónico. Concepción: Universidad de Concepción.

Gehl, J. (2006). La humanización del espacio urbano. La vida social entre los edificios. Estudios universitarios de arquitectura. Barcelona: Editorial Reverté.

Gibbs, G. (2012). El análisis de datos cualitativos en investigación cualitativa. Barcelona: Ediciones Morata.

Lefebvre, H. (1 976). Espacio y política. El derecho a la ciudad II. Barcelona: Ediciones Península.

MINVU. (2009). Espacios públicos. Recomendaciones para la gestión de proyectos. Santiago: Ministerio de Vivienda y Urbanismo, División de Desarrollo Urbano. Impresión Maval.
Muñoz, M. D. (1995). Estudio del patrimonio en la Universidad de Concepción. Plan Regulador del Campus. Tomo 1. Memoria General. Concepción: Dirección de Planificación e Informática. Universidad de Concepción.

Rodríguez L. y Carrasco, B. (2016). Lugares con sentido, identidad y teoría urbana: Los casos de las ciudades de Concepción y Talca. Revista de Geografía Norte Grande, (64), 167-186.

Schlack, E. (2007). Espacio Público. ARQ (Santiago), (65), 25-27.

Torres, C. (2015). El campus universitario de la Universidad de Concepción en el contexto Latinoamericano (Tesis doctoral). Universidad de Sevilla. Departamento de Historia, Teoría y Composición Arquitectónica, Concepción. 